\title{
Clinical audit of breast cancer care at the Benavides Cancer Institute, University of Santo Tomas Manila, Philippines: Part I, methods and tools
}

This article was published in the following Dove Press journal:

Clinical Audit

5 January 2015

Number of times this article has been viewed

Teresa T Sy-Ortin'

Michael Benedict A Mejia'

Janine Margarita Dizon ${ }^{1-3}$

Karen Grimmer ${ }^{2}$

Consuelo Gonzalez-Suarez ${ }^{2,3}$

Warren Bacorro'

Jayson $\mathrm{Co}^{\prime}$

Priscilla Caguioa ${ }^{4}$

Clevelinda Calma ${ }^{4}$

Eugenio Regala ${ }^{4}$

Kathleen Baldivia'

Rowen Yolo ${ }^{5}$

Karl Morales ${ }^{6}$

Josefino Sanchez ${ }^{6}$

Ray Malilay ${ }^{6}$

Ida Marie Tabangay-Lim ${ }^{6}$

Jocelyn C Que 7,8

Mary Jocylyn Bautista ${ }^{7}$

'Department of Radiation Oncology, Benavides Cancer Institute, University of Santo

Tomas Hospital, Espana, Manila, Philippines;

${ }^{2}$ International Centre for Allied Health Evidence, University of South Australia, Adelaide, SA,

Australia; ${ }^{3} \mathrm{Centre}$ for Health Research and

Movement Science, University of Santo

Tomas, Espana, Manila, Philippines; ${ }^{4}$ Section of

Medical Oncology, Department of Medicine,

University of Santo Tomas Hospital, Espana,

Manila, Philippines; ${ }^{5}$ Department of Pathology,

University of Santo Tomas Hospital, Espana,

Manila, Philippines; ${ }^{6}$ Department of Surgery,

University of Santo Tomas Hospital, Espana,

Manila, Philippines; 'Pain Management and

Palliative Care Unit, Benavides Cancer Institute,

University of Santo Tomas Hospital, Espana,

Manila, Philippines; ${ }^{8}$ Center for Pain Medicine,

Faculty of Medicine and Surgery, University of

Santo Tomas, Espana, Manila, Philippines

On behalf of the Benavides Cancer Institute Breast Cancer Working Group (BCIBC-WG)

Correspondence: Janine Margarita Dizon University of Santo Tomas Hospital, Manila, Philippines

Tel +632406 I6I I extn 4035 or 8454

Fax +632740 97।3

Email jmrdizon@yahoo.com

Jayson Co

University of Santo Tomas Hospital, Manila,

Philippines

Tel +63922575 5558

Email jc_polar@yahoo.com
Background: The prevalence and incidence of breast cancer has been increasing worldwide. It has been reported that the Philippines has the highest number of cases in Asia, and breast cancer is now the leading cause of death in the country. This study protocol presents the methodological plan for a quality improvement study that will assess the current practice of breast cancer examination, diagnosis, and management at one of the leading cancer institutions in the Philippines, the University of Santo Tomas Hospital-Benavides Cancer Institute (USTH-BCI); and map with standards of care, in order to identify areas that would need improvement to facilitate best practice care for breast cancer patients.

Methods: This study has been approved by the Institutional Review Board of the University of Santo Tomas Hospital. A breast cancer working group has been established (Benavides Cancer Institute Breast Cancer Working Group [BCIBC-WG]) during a 1 day meeting of cancer specialists at USTH-BCI. The meeting was facilitated by both international and local methodologists in the field of evidence-based practice and quality improvement. A quality improvement plan and a clinical audit protocol for assessing current practice were drafted during this meeting. The clinical audit of current breast cancer care will be undertaken at USTH-BCI using medical records review. Clinical indicators of outcomes were identified and typical patient journeys were mapped to develop the data collection/extraction form. The data collection forms were sent to experts for face and content validation, to ensure a valid and comprehensive collection of the data. The form was revised as needed. Three hundred and eighteen (318) breast cancer cases were seen at USTH-BCI in the year 2012, and all 318 records will be reviewed as decided by the group. A reliability procedure will be undertaken among data collectors of the study and pilot testing procedure will be undertaken to test the feasibility of the data collection methods. Data will be analyzed and reported using means and percentages as appropriate. Missing data will also be reported in order to identify strategies to ensure completeness of medical records in the future.

Keywords: breast cancer, audit, quality improvement, developing countries, protocol

\section{Background}

The increasing prevalence and incidence of cancer worldwide is alarming. In the United States alone, the prevalence of cancer in 2011 was 12,549,000. ${ }^{1}$ In less developed countries, the incidence of cancer is projected to increase from $56 \%$ of world cases in 2008 to greater than $60 \%$ by 2030 . Cancer is also the leading cause of death globally. The World Health Organization reports that the number of deaths due to cancer is projected to increase by $45 \%$ from 2007 to 2030 (from 7.9 million to 11.5 million deaths). ${ }^{2}$

With the alarming increase in the prevalence and incidence of cancer and the rate of mortality due to cancer worldwide, much research has been done on identification 
of risk factors, prevention and management. Such that, with advancements in medical science research, one third of cases can now be prevented, one third can be diagnosed early and thus managed early, and one third can be treated. ${ }^{3}$ However, this is only possible if management and treatment provided are consistent with evidence-based standards of cancer care. ${ }^{3}$

In the Philippines, cancer was one of the leading causes of morbidity and mortality in 2002: the leading cancer types are lung, breast, cervix, liver and colon. ${ }^{4}$ More recently, the Philippines' Department of Health and the Philippine Cancer Society have reported that breast cancer is now the leading cancer type in the country and accounts for the highest incidence rate in Asia. ${ }^{5}$ Thus, the Philippines' Department of Health, in partnership with the Philippine Cancer Society has developed programs in relation to screening, prevention, and management of cancer. ${ }^{6}$ Programs such as free screening, medicines for poor women diagnosed early with breast cancer, and the " $Z$ Benefit Package of PhilHealth" were developed and implemented to address the alarming increase in breast cancer morbidity and mortality. ${ }^{6}$

The University of Santo Tomas Hospital (USTH), one of the leading private health institutions in the Philippines, established the Benavides Cancer Institute (BCI) in 2006. The mission of the USTH-BCI is to strengthen the national cancer care program and provide comprehensive and multidisciplinary cancer care for diagnosis, treatment and prevention. ${ }^{7}$ Consistent with its mission, the USTH-BCI is continuously planning and strategizing ways to identify and deliver the best cancer care in the Philippines.

As medical science research has reported that cancer can be prevented, diagnosed early, and treated if care management and treatment programs are consistent with evidence-based guidelines, the USTH-BCI developed a research project that will assess current practice and then map current practice with existing evidence-based guidelines for breast cancer care such as the National Comprehensive Cancer Network (NCCN) Guidelines for breast cancer. ${ }^{8}$ USTH-BCI agreed to use the NCCN guidelines, ${ }^{3}$ as standard of care to underpin health care provided to patients. However, it is not known whether the standards of care are being adhered to or complied with by all health professionals involved in the care of breast cancer patients. There is much discussion in the literature regarding the availability of evidence-based standards of care and the existing gap in compliance with the standards..$^{9-11}$ For instance, whilst guideline adherence was associated with improved survival, one study reports that guideline adherence was low for patients with triple negative breast cancer. ${ }^{9}$ Another one reports less than half of women with breast cancer completed the recommended therapy. ${ }^{12}$ Thus, it is very important to assess whether existing practice follows the recommended standards, and then later, plan for strategies that would address the gaps and plan for sustainable efforts in the long term to improve clinical outcomes of care.

This may be an ambitious project but it could be a milestone in clinical practice and health care as this is the first attempt to evaluate current practices in breast cancer care in the Philippines. This is valuable because identifying current practices allows: 1) identification of relevant data items to be used as basis for assessing breast cancer care delivery; 2) identification of gaps which are useful in identifying areas which need improvement; 3) opportunities for better patient outcomes and better utilization of resources, especially in developing countries, such as the Philippines, with limited resources and lastly; 4) change and improve health policies in order to standardize health services and improve the health system, thus, championing best breast cancer care in the Philippines. ${ }^{13,14}$

\section{Objectives}

This study protocol presents the methods for undertaking a quality improvement project that will describe the current practice of breast cancer examination, diagnosis, and management at the USTH-BCI, and map whether current practice is consistent with evidence based standard of care.

\section{Methods}

\section{Ethics}

Ethical approval was obtained from the Institutional Review Board of the USTH, Manila, Philippines.

\section{Study design and setting}

A clinical audit study using medical record review will be undertaken to address the study objectives. The clinical audit will be undertaken at the USTH-BCI.

\section{Reference population}

Patients with breast cancer seen at USTH-BCI can come from any of the following:

1. Patients in the clinical division of the USTH and referred to BCI for screening/evaluation, diagnosis/staging and treatment and management

2. Patients seen by a medical doctor within USTH and referred to BCI for diagnosis/staging and then for treatment and management

3. Patients diagnosed elsewhere in the Philippines and referred for breast cancer treatment and management from other institutions in the Philippines. 
Patient with mammogram findings suspecting malignancy OR any of the following symptoms:
1. Breast mass
2. Breast asymmetry
3. Nipple discharge
4. Nipple bleeding
5. Nipple retraction
6. Skin dimpling
7. Breast pain
8. Axillary mass

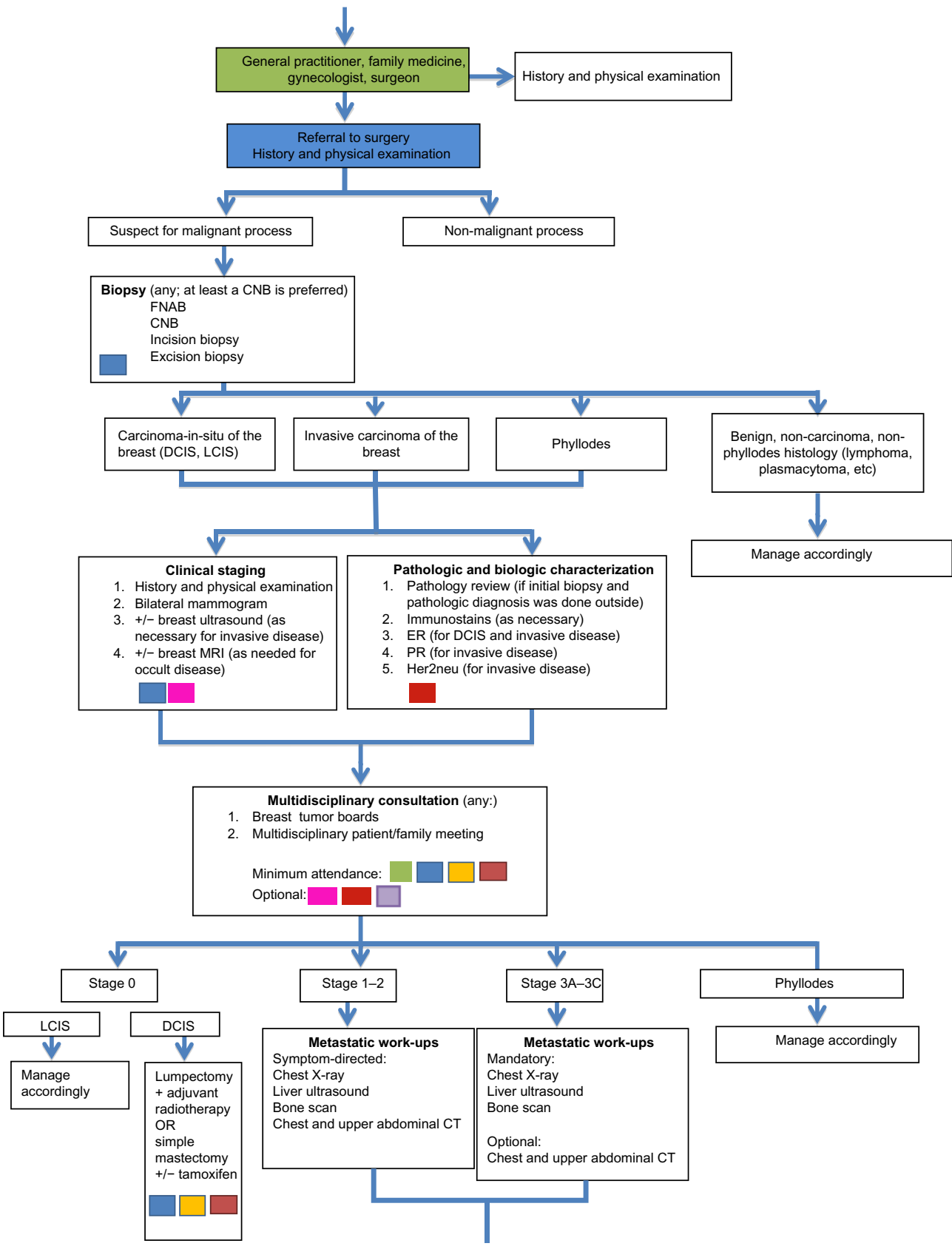

Figure I (Continued) 


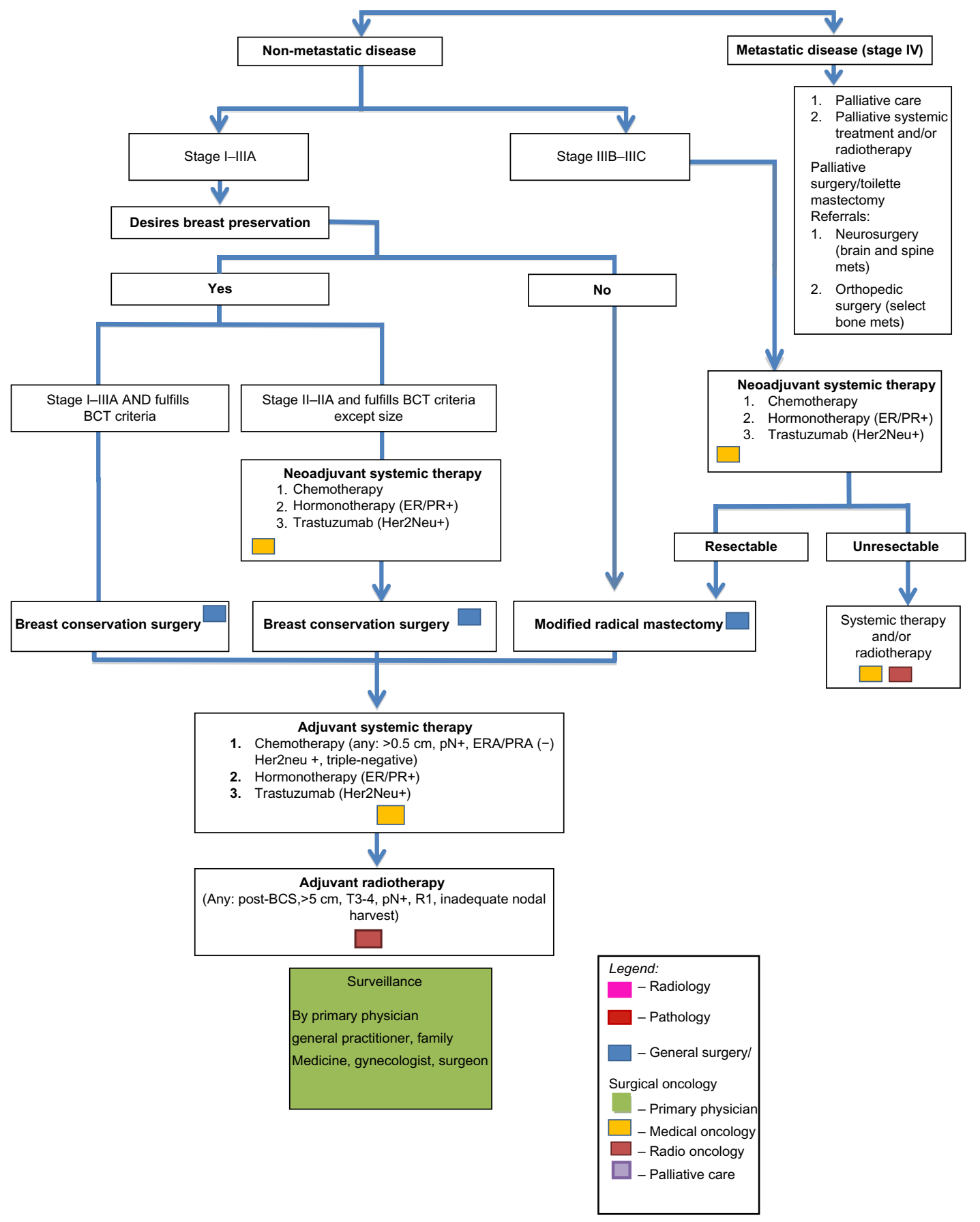

Figure I Typical patient journey of patients with breast cancer.

Note: Patients may be referred to USTH-BCl at any point in the patient journey.

Abbreviations: USTH-BCI, University of Santo Tomas Hospital-Benavides Cancer Institute; LCIS, lobular carcinoma in situ; MRI, magnetic resonance imaging; Her2neu, human epidermal growth factor receptor 2; CT, computed tomography; Mets, metastasis; BCT, breast conservation therapy; CNB, core needle biopsy; ER, estrogen receptor; PR, progesterone receptor; FNAB, fine needle aspiration biopsy; DCIS, ductal carcinoma in situ.

\section{Sampling method and sample size}

A total of 318 patients with breast cancer were seen at BCI for the year 2012. As 318 is a manageable number of breast cancer patient cases, the group decided to consider all these cases for inclusion in the audit, thus using census sampling. This will allow $100 \%$ statistical power and accuracy in describing the current practice in different categories of breast cancer care provided by BCI. ${ }^{15}$

\section{Working group}

The clinical audit proposal was formulated by the BCI Breast Cancer Working Group (BCIBC-WG) consisting of 
key health care personnel involved in breast cancer care at USTH-BCI (Teresa SyOrtin, MD, chair of BCI; Priscilla B Caguioa, MD; Clevelinda Calma, MD; Eugene Regala, MD; Kathleen Baldivia, MD; Rowen Yolo, MD; Michael A Mejia, MD; Karl Morales, MD; Josefino Sanchez, MD; Ray Malilay, MD; Ida Marie Tabangay-Lim, MD; Jocelyn Que, MD; Joycylyn Bautista, MD; Warren Bacorro, MD; and Jayson L Co, MD; and methodologists in the area of quality improvement and evidence based practice, (JRD, the project leader; $\mathrm{KG}$, an external collaborator; and CGS, a local evidence based practice [EBP] champion). The

Table I Profile of breast cancer patients seen at USTH-BCI

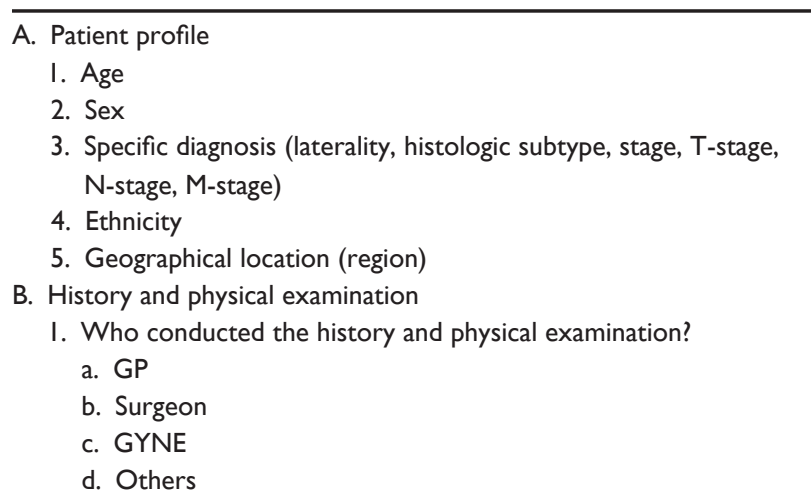

2. Length of referral from the attending physician to the surgeon a. $<$ I week

b. $>$ I week

c. $>$ I month

d. Others

And from which institution was the patient coming from

3. Elements in the history taking

a. Family history of CA

b. OB history

I. menstrual history

2. hormonal therapy

3. parity

4. others

c. Previous history of mammograms (number and results)

d. Previous surgeries

e. Other malignancies

4. Physical examination

a. Description of breast mass

I. size

2. laterality

3. quadrant

4. clock position

5. distance from the nipple $(\mathrm{cm})$

6. skin changes

7. mobility

b. Axillary nodes (if palpable, number and mobility)

c. Supraclavicular area

d. Chest PE (auscultatory findings)

e. Abdominal PE (liver, spleen)

Abbreviations: USTH-BCl, University of Santo Tomas Hospital-Benavides Cancer Institute; GP, General Practitioner; GYNE, gynecologist; CA, cancer; OB, obstetrics and gynecology; PE, physical examination.
BCIBC-WG held a 1 day meeting on quality improvements and audits, role of clinical guidelines in improving quality of health care services, and planning the quality improvement proposal of BCI.

\section{Identification of patient journeys}

In order to identify the current practices in breast cancer care at BCI a typical patient journey was identified by the BCIBC-WG. Patient journeys are visualizations of the usual flow of relevant processes a patient undergoes when seen in the facility. ${ }^{16}$ A copy of the patient journey was then sent to other health care personnel involved in breast cancer care for validation of the processes. Thus, the patient journey presented in Figure 1 has been validated by all involved in breast cancer care at BCI and will be the basis of this clinical audit.

\section{Audit data items}

The BCIBC-WG has identified an agreed list of items to be included in the clinical audit to obtain a comprehensive profile of breast cancer care at BCI (Tables 1-3).

\section{Data collection methods Data collection tool}

A standard data collection form (Supplementary materials) will be used to retrieve the relevant audit items from the

Table 2 Clinical audit of current practice on diagnosing/staging of patients with breast cancer at USTH-BCl

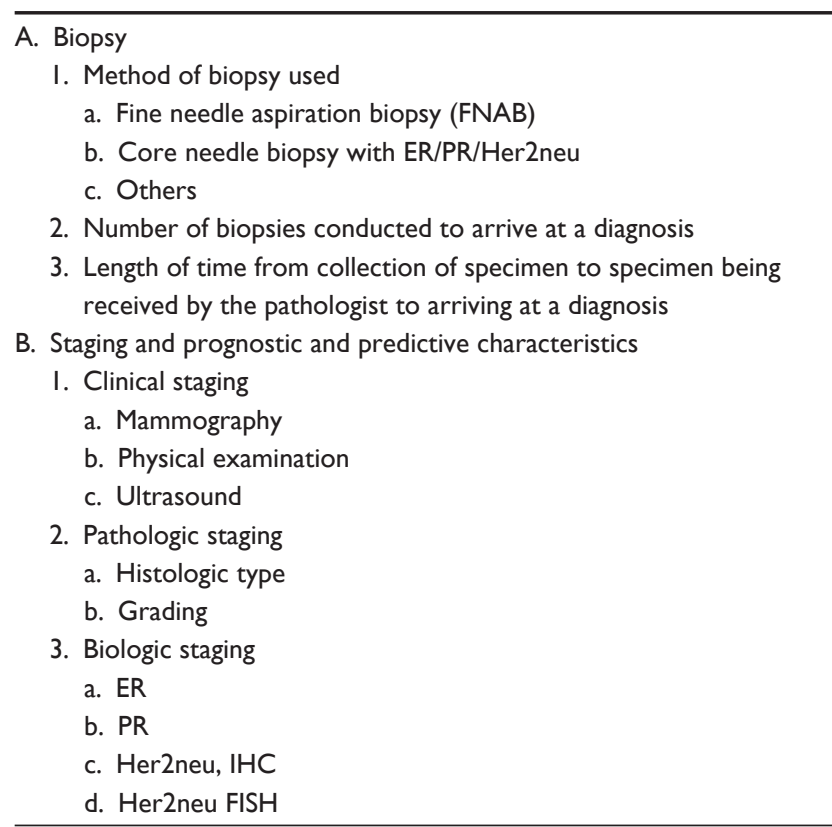

Abbreviations: USTH-BCl, University of Santo Tomas Hospital-Benavides Cancer Institute; ER, estrogen receptor; PR, progesterone receptor; Her2neu, human epidermal growth factor receptor 2; IHC, immunohistochemistry; FISH, fluorescence in situ hybridization. 
Table 3 Clinical audit of treatment and management of patients with breast cancer referred to USTH-BCI

A. Date since first diagnostic exam to start of treatment

B. Multidisciplinary consultation

I. Breast tumor boards

2. Multidisciplinary patient/family meeting

3. Specialty disciplines attending the multidisciplinary consultation

C. Stages of breast cancer: 0, I, 2, 3, 4

D. Stage 0

I. LCIS or DCIS

2. If LCIS, procedures

a. Genetic counselling

b. Surveillance

c. Chemo prevention

d. Prophylactic surgery

3. If DCIS,

a. Surgical procedure

I. Lumpectomy

2. Axillary lymph nodes dissection

3. Simple mastectomy

4. Modified radical mastectomy

b. Adjuvant therapy given

c. Tamoxifen given

E. Stage I and 2 by PE

I. Symptom metastatic work ups done

F. Stage 3 by PE

I. Mandatory tests done

a. Chest X-ray

b. Liver ultrasound

c. Bone scan

2. Optional tests done

a. Chest and upper abdominal CT

3. Appropriate surgical management given

a. Fulfills BCT criteria

b. Preference and reason for preference

4. Surgical procedure done (BCS or MRM)

5. If $B C S$,

a. Pre-BCS treatment

I. Chemotherapy

2. Hormonotherapy

3. Trastuzumab

b. Adjuvant systemic therapy

I. Chemotherapy

2. Hormonotherapy

3. Trastuzumab

c. Adjuvant radiotherapy

6. If MRM,

a. Indications noted $(>0.5 \mathrm{~cm}, \mathrm{pN}+$, ER/PR (-), Her2neu+, triple negative)

b. Adjuvant systemic therapy

I. Chemotherapy

2. Hormonotherapy

3. Trastuzumab

d. Adjuvant radiotherapy

G. Clinical stage IIIB-IIIC

I. Neoadjuvant systemic therapy

I. Chemotherapy

2. Hormonotherapy

3. Trastuzumab
Table 3 (Continued)
2. Surgery
a. Adjuvant systemic therapy
I. Chemotherapy
2. Hormonotherapy
3. Trastuzumab
b. Adjuvant radiotherapy
3. If metastatic, referral to palliative care
4. Other treatment options
5. If with brain metastasis, referral to neurosurgeon
I. Referral to palliative care
2. Referral for other options
I. Managed accordingly
J. Surveillance
I. Date since surgery
2. Date since last chemotherapy/adjuvant therapy
3. Follow-up date:
No evidence of disease
Local recurrence
Local recurrence/distant metastases
Distant metastases

H. Stage 4

I. Phyllodes

Abbreviations: USTH-BCl, University of Santo Tomas Hospital-Benavides Cancer Institute; LCIS, lobular carcinoma in situ; DCIS, ductal carcinoma in situ; $\mathrm{PE}$, physical examination; $\mathrm{CT}$, computed tomography; $\mathrm{BCT}$, breast conservation therapy; BCS, breast conservation surgery; MRM, modified radical mastectomy; ER, estrogen receptor; PR, progesterone receptor; Her2neu, human epidermal growth factor receptor 2 .

patients' medical records. This form includes information from patient demographics, history and physical examination, diagnosis/stages of breast cancer and management for breast cancer patients. Consent from the attending physicians will be obtained.

\section{Validity of the data collection tool}

The data collection form was sent to experts (surgeon, a medical oncologist and a pathologist) and comments were discussed in a meeting for face and content validation. This was to ensure that the data audit items to be retrieved from the medical records will answer the clinical audit objectives. Revisions were undertaken based on the validation procedure and sent back to the experts for approval.

\section{Pilot testing the audit data collection process}

The clinical audit data collection will be pilot tested to ensure validity and reliability of the procedures. ${ }^{15,17}$ More specifically, the purposes of the pilot testing are as follows:

1. Test feasibility of the data collection methods

2. Estimate the amount of time and resources required to collect data

3. Check data for completeness. 
Table 4 Quality indicators in the assessment of breast cancer care

Quality indicators
Diagnosis
Completeness of clinical and
imaging diagnostic work-up
(percentage)
Completeness of prognostic/predictive
characterization

\section{Data elements}

Proportion of patients who preoperatively underwent:

I. Mammography

2. Physical examination

3. Biopsy

4. Ultrasound

Proportion of patients (invasive cancer cases) for which the following prognostic/predictive parameters have been recorded:

I. Histologic type

2. Grading

3. Her2 testing

Proportion of patients (invasive cancer cases) with primary surgery for which the following prognostic/ predictive parameters have been recorded:

I. Histologic type

2. Grading

3. $E R$

4. Her2 testing

5. Pathologic testing ( $\mathrm{T}$ and $\mathrm{N}$ )

6. Size in $\mathrm{mm}$ for invasive component

7. Peritumoral vascular invasion

8. Distance to nearest radial margin

Proportion of patients (non-invasive cancer cases) for which the following prognostic/predictive parameters have been recorded:

I. Dominant histologic pattern

2. Size in $\mathrm{mm}$ (best pathology or radiology estimate if 2 stage pathology)

3. Grading

4. Distance to nearest radial margin

Waiting time

Surgery and loco-regional treatment Multidisciplinary discussion

Appropriate surgical approach

Postoperative RT

\section{Avoidance of over treatment \\ Overtreatment}

\section{Systemic treatment}

Appropriate hormonotherapy

Appropriate chemotherapy and other medical therapy

\section{Staging, counseling, follow-up and rehabilitation}

Appropriate staging procedure

Appropriate staging procedure

Appropriate follow-up tumor
Date from first diagnostic examination to date of surgery or first treatment

Proportion of cancer patients to be discussed by a multidisciplinary team

Proportion of patients (invasive cancer cases) who received a single (breast) operation for the primary

Proportion of patients (invasive cancer cases) who received postoperative RT after surgical resection of primary tumor and appropriate staging/surgery

Proportion of patients with involvement of axillary lymph nodes $(>p N 2 a)$ who received postmastectomy radiotherapy

Proportion of patients with invasive breast cancer not $>3 \mathrm{~cm}$ who underwent BCT

Proportion of patients with non-invasive breast cancer not $>2 \mathrm{~cm}$ who underwent BCT

Proportion of patients with endocrine sensitive invasive carcinoma who received hormonotherapy, out of those with diagnosis

Proportion of patients with ER- and PR-carcinoma who received did not receive adjuvant hormonotherapy, out of those with diagnosis

Proportion of patients with ER- ( $T>$ I cm or Node+) invasive carcinoma who received adjuvant chemotherapy, out of those with diagnosis

Proportion of patients with $\mathrm{N}+$ or $\mathrm{N}-\mathrm{T}>\mathrm{I} \mathrm{cm}$ Her2neu+ (IHC3+ or +FISH) invasive carcinoma treated with chemotherapy and had adjuvant trastuzumab out of those with same diagnosis

Proportion of women with stage I breast cancer who do not undergo metastatic staging tests

Proportion of women with stage 3 breast cancer who undergo metastatic staging tests

Proportion of asymptomatic patients who undergo routine annual mammographic screening and clinical evaluation every 6 months in the first 5 years after the operation

Proportion of patients undergoing periodic history taking, physical examination and annual mammography

Abbreviations: Her2, human epidermal growth factor receptor 2; ER, estrogen receptor; RT, radiotherapy; BCT, breast conservation therapy; PR, progesterone receptor; $\mathrm{IHC}$, immunohistochemistry 3; FISH, fluorescence in situ hybridization. 


\section{Data collectors}

Two to three data collectors will be identified to retrieve the audit items from the medical records. They should have experience in data recording or management to ensure ability to reliably extract the information needed. An orientation to the clinical audit project and training for data collection will be conducted with the data collectors. A reliability procedure will also be undertaken by asking the data collectors to complete data extraction of five sample cases independently. The inter-rater reliability for percentage of agreement recommended by Dixon and Pearce will be used to compute the reliability of the data collectors. ${ }^{15}$ This is done by dividing the number of bits of data for which there was complete agreement among the data collectors and the total number of bits of data (for example, 25 bits of data per case $\times 5$ cases). Further training will be provided as needed based on the results of the reliability procedure.

\section{Data handling}

A purpose built MSExcel file will be constructed, which restricts the type of data which can be entered into each column. This will reduce data entry errors and ensure efficiency of data amalgamation. All patient cases to be included in the clinical audit will be provided with a code. This code will be used and entered as the patient case in the MSExcel file including all audit items retrieved from the patient medical records.

\section{Auditing guidelines}

Table 4 lists the quality indicators that will be used later to evaluate the outcome of current practice (relevant items extracted from the European Society of Breast Cancer Specialists Quality indicators in breast cancer position paper by Del Turco et al). ${ }^{18}$

\section{Data analysis}

This will be undertaken by an independent statistician. Data will be reported using means and percentages as appropriate. Missing data will also be reported in order to identify strategies to ensure completeness of medical records in the future particularly when assessing adherence to guidelines.

\section{Confidentiality}

All data to be obtained from the medical records shall be kept confidential and will only be available to the working group.

\section{Disclosure}

The authors have no conflicts of interest to declare.

\section{References}

1. Howlader N, Noone AM, Krapcho M, et al. SEER cancer statistics review, 1975-2009 (vintage 2009 populations). Bethesda, MD: National Cancer Institute (2012).

2. World Health Organization [homepage on the Internet]. Available from http://www.who.int/features/qa/15/en/index.html. Accessed April 17, 2013.

3. World Health Organization [homepage on the Internet]. Available from http://www.who.int/mediacentre/factsheets/fs297/en/index.html. Accessed April 17, 2013.

4. Ngelangel C, Wang E. Cancer and the Philippine Cancer Control Program. J JClin Oncol. 2002;32(1):S52-S61.

5. Philippine Council for Health Research and Development. The ABCs of breast cancer. [webpage on the Internet]. Available from http://www. pchrd.dost.gov.ph/index.php/2012-05-23-07-46-36/2012-05-24-00-0306/5689-the-abcs-of-breast-cancer. Accessed April 17, 2013.

6. Philippines' Department of Health. Available from http://www. philhealth.gov.ph/news/2013/zbenefits_saves.html. Accessed April 20, 2013.

7. The Varsitarian. Available from http://www.varsitarian.net/news/ cancer_institute_launched. Accessed April 20, 2013.

8. National Comprehensive Cancer Network guidelines for treatment of breast cancer. Available from https://www.nccn.org/store/login/login. aspx?ReturnURL=http://www.nccn.org/professionals/physician_gls/ pdf/breast.pdf. Accessed May 30, 2014.

9. Schwentner L, Wöckel A, König, et al, for the Brenda study group. Adherence to treatment guidelines and survival in triple-negative breast cancer: a retrospective multi-center cohort study with 9156 patients. BMC Cancer. 2013;13:487.

10. Chagpar R, Xing Y, Chiang Y, Feig W, Chang G, You N, et al. Adherence to stage-specific treatment guidelines for patients with colon cancer. J Clin Oncology. 2012;30(9):972-979.

11. Barni S, Venturini M, Molino A, et al. Importance of adherence to guidelines in breast cancer clinical practice. The Italian experience (AIOM). Tumori. 2011;97:559-563.

12. Hershman D, Kushi L, Shao T, et al. Early discontinuation and nonadherence to adjuvant hormonal yherapy in a cohort of 8,769 early-stage breast cancer patients. J Clin Oncology. 2010;28(27):4120-4128.

13. Wensing $M$, van der Weijden T, Grol R. Implementing guidelines and innovations in general practice: which interventions are effective? Brit J Gen Pract. 1998;48:991-997.

14. Grol R, Grimshaw J. From best evidence to best practice: effective implementation of change in patients' care. The Lancet. 2003;362: $1225-1230$.

15. Dixon N, Pearce M. Clinical Audit Manual. Romsey: Healthcare Quality Quest Ltd; 2010.

16. National Health Survey. Available from http://www.institute.nhs. uk/quality_and_service_improvement_tools/quality_and_service_ improvement_tools/process_mapping_-_an_overview.html Accessed April 23, 2013.

17. Banks NJ. Designing medical record abstraction forms. Int J Qual Health Care. 1998;10(2):163-167.

18. Del Turco M, Ponti A, Bick U, et al. Quality Indicators in breast cancer care. Eur J Cancer. 2010:2344-2356. 


\section{Supplementary material}

\section{Benavides Cancer Institute's Breast Cancer Audit Form}

\section{PATIENT PROFILE}

A. Profile

Patient ID No.

1. Name:

2. Age:

3. Gender:

[ ] Female [ ] Male

4. Final pathologic diagnosis:

- Laterality [ ] Right [ ] Left [ ] Bilateral

- Histologic subtype [ ] IDC [ ] ILC [ ] Other

- Stage [ ] 0 [ ] I [ ] IIA [ ] IIB [ ] IIIA [ ] IIIB [ ] IIIC [ ] IV

- T-stage [ ] is [ ] 1 [ ] 2 [ ] 3 [ ] 4

- N-stage [ ] 0 [ ] 1 [ ] 2 [ ] 3

- M-stage [ ] 0 [ ] 1

5. Ethnicity:

6. Geographical Location (Region) by residence:

[ ] NCR [ ] CAR [ ] ARM

[ ] 1 [ ] 2 [ ] 3 [ ] 4-A [ ] 4-B [ ] 5 [ ] 6

[] 7 [ ] 8 [] 9 [] $10[$ [ ] 11 [ ] 12 [] 13

\section{B. History and physical examination}

1. Who conducted the history taking?

[ ] General Physician [ ] Surgeon [ ] Gynaecologist [ ] Others:

[] Specialist [ ] Trainee

2. Length of referral from the physician who took the history to the surgeon

[ ] 1 week [ ] $>1$ week [ ] $>1$ month [ ] others:

Reasons for delay:

From which institution:

3. Elements of the history taking

a. Family history of cancer

1. [ ] With history OR [ ] Without history

If with history, identify the site/s:

a.

b.

c.

b. OB history

1. [ ] With menstrual history OR [ ] Without menstrual history

2. [ ] Hormone use OR [ ] No hormone use

If+ hormone use, identify the type:

3. Parity

[ ] 0 [ ] 1 [ ] 2 [ ] 3 [ ] 4 [ ] 5 [ ] 6 [ ] 7 [ ] 8 [ ] 9 [ ] 10 [ ] > 10

4. Others:

c. Previous history of mammogram

1. [ ] With mammogram OR [ ] Without mammogram 
If with mammogram, number of mammograms done:

[] $0[$ [ ] 1[] $2[$ [ 3

2. Results of mammogram and identify classification checklist used:

d. Previous history of surgery:

1. [ ] With history of surgery OR [ ] Without history of surgery

If with history of surgery, identify:

a.

b.

c.

e. Other malignancy

1. [ ] With other malignancy OR [ ] Without other malignancy If with other malignancy, identify:

a.

b.

c.

4. Elements of physical examination

a. Description of breast mass

1. Size of breast mass: $(\mathrm{cm})$

2. Laterality: [ ] Right [ ] Left

3. Quadrant: [ ] Upper outer quadrant, [ ] Upper inner quadrant, [ ] Lower outer quadrant, [ ] Lower inner quadrant

4. Clock position

[ ] 3:00 o'clock [ ] 6:00 o'clock [ ] 9:00 o'clock [ ] 12:00 o'clock

5. Distance from the nipple $(\mathrm{cm})$ :

6. Skin changes:

[ ] With skin changes OR [ ] Without skin changes

7. Mass mobility:

[ ] Fixed OR [ ] Mobile

b. Presence of nodes:

[ ] With axillary nodes OR [ ] With supraclavicular nodes

If with nodes: number of nodes: mobility:

c. Chest PE:

d. Abdominal PE:

\section{DIAGNOSIS AND STAGING}

\section{A. Biopsy}

1. Method of biopsy used

[ ] FNAB [ ] Core needle biopsy with ERA/PRA/Her2neu [ ] others

2. Number of biopsies to arrive at a diagnosis:

3. Date of collection of specimen:

4. Date of specimen received at pathology laboratory:

5. Date of diagnosis:

\section{B. Staging and prognostic and predictive characteristics}

\section{Clinical staging}

[ ] Mammography [ ] Physical examination [ ] Ultrasound

2. Pathologic staging

[ ] Histologic type [ ] Grading

3. Biologic staging

[ ] ERA [ ] PRA [ ] Her2neu, iHC [ ] Her2neu FISH 


\section{MANAGEMENT OF BREAST CANCER}

\section{A. Multidisciplinary consultation:}

1. Multidisciplinary consultation done [ ] Yes OR [ ] No

2. Type: [ ] breast tumor boards [ ] multidisciplinary patient/family meeting

3. Attended by:

\section{B. Stage of breast cancer:}

1. Stage 0: [ ] LCIS OR [ ] DCIS

a. If LCIS, what was done:

[ ] genetic counselling [ ] surveillance [ ] chemo prevention [ ] prophylactic surgery

b. If DCIS, what surgical procedure was done; check any that applies:

[ ] lumpectomy [ ] axillary lymph node dissection [ ] simple mastectomy [ ] MRM

c. Was adjuvant radiotherapy given, [ ] Yes [ ] No

d. Was tamoxifen given, [ ] Yes [ ] No

2. Stage 1 and 2 by PE

a. Symptom directed metastatic work ups:

[] chest x-ray

[ ] liver ultrasound

[ ] bone scan

[ ] chest CT

[ ] upper abdominal CT

[ ] others, specify:

If metastatic proceed to

If non-metastatic, proceed to Clinical stage I-IIIA (M0 on work up)

3. Stage 3 by PE

a. Mandatory:

[ ] Chest x-ray

[ ] Liver ultrasound

[] Bone Scan

b. Optional:

[ ] Chest and upper abdominal CT

If metastatic proceed to

If non-metastatic IIIA, proceed to Clinical stage I-IIIA (M0 on work up)

If non-metastatic IIIB-IIIC, proceed to Clinical stage IIIB-IIIC (M0 on work up)

c. Appropriateness of surgical management:

1. Fulfils BCT criteria: [ ] Yes [ ] No, [ ] Yes, except size

2. Preference: [ ] BCT [ ] MRM, reason:

d. Surgical procedure: [ ] BCS [ ] MRM

If BCS,

1. Was pre-BCS systemic treatment given: [ ] Yes [ ] No

If Yes, check any that applies:

[ ] chemotherapy

[ ] hormonotherapy

[ ] trastuzumab

2. Was adjuvant systemic therapy given: [ ] Yes [ ] No

If YES, check any that applies:

[ ] chemotherapy

[ ] hormonotherapy

[ ] trastuzumab 
3. Was adjuvant radiotherapy given: [ ] Yes [ ] No

If MRM,

1. Indications for adjuvant systemic therapy, check all that apply:

[ ] $>0.5 \mathrm{~cm}$

[ ] $\mathrm{pN}+$

[ ] ERA/PRA(-)

[ ] Her2neu +,

[ ] triple-negative

2. Adjuvant systemic therapy given: [ ] Yes [ ] No

If YES, check any that applies:

[ ] chemotherapy

[ ] hormonotherapy

[ ] trastuzumab

3. Was adjuvant radiotherapy given: [ ] Yes [ ] No

4. Clinical stage IIIB-IIIC

a. Was neoadjuvant systemic therapy given [ ] Yes [ ] No

If YES, check any that applies:

[ ] chemotherapy

[ ] hormonotherapy

[ ] trastuzumab

b. Was surgery done [ ] Yes [ ] No

If surgery was done, was adjuvant systemic therapy given: [ ] Yes [ ] No

If YES, check any that applies:

[ ] chemotherapy

[ ] hormonotherapy

[ ] trastuzumab

c. Was adjuvant radiotherapy given: [ ] Yes [ ] No

5. If metastatic, was palliative care referral done [ ] Yes [ ] No

a. Were other treatment options aside from palliative care given [ ] Yes [ ] No

Palliative systemic treatment

[ ] chemotherapy

[ ] hormonotherapy

[ ] trastuzumab

[ ] bone directed

[ ] radiotherapy

[ ] palliative surgery

[ ] toilette mastectomy

b. If with brain metastasis, was referral to neuro surgeon given [ ] Yes [ ] No

Clinical Audit

\section{Publish your work in this journal}

Clinical Audit is an international, peer-reviewed, open access journal focusing on the processes and outcomes of clinical audit in any area of healthcare. All aspects of patient care are addressed within the journal and practitioners from all disciplines are invited to submit their work. Areas covered include: Publication of audits; How an audit has changed practice;

\section{Dovepress}

Practical tips on how to do audits and to avoid pitfalls; How audits have changed patient care; Calls and justifications for new audits. The manuscript management system is completely online and includes a very quick and fair peer-review system, which is all easy to use. Visit http://www.dovepress. com/testimonials.php to read real quotes from published authors. 